that alcohol abuse was not related to impairments in daily functioning (assessed with the World Health Organization Disability Assessment Schedule II) and had a favourable course (remission rate after 2 years: 93.5\%). Apparently, alcohol abuse is characterised by only minimal alcohol-related impairments, which may clarify our finding that it was not related to the persistence of depression and anxiety. Note that alcohol abuse in our sample of out-patients with depression or anxiety or in the general population might differ substantially from alcohol abuse in clinical samples of severe abusers with regard to associated levels of impairment.

Taken together, these findings emphasise the importance of severity indicators, rather than dichotomous diagnoses of alcohol dependence or abuse, in the assessment of alcohol problems. This is in line with the proposal of the DSM-5 Work Group to distinguish different levels of severity within the diagnosis of an alcohol use disorder (www.dsm5.org).

1 Boschloo L, Vogelzangs $\mathrm{N}$, Van den Brink W, Smit JH, Veltman DJ, Beekman ATF, et al. Alcohol use disorders and the course of depressive and anxiety disorders. Br J Psychiatry 2012; 200: 476-84.

2 World Health Organization. Global Status Report on Alcohol and Health. WHO 2011.

3 Haynes JC, Farrell M, Singleton N, Meltzer H, Araya R, Lewis G, Wiles NJ. Alcohol consumption as a risk factor for anxiety and depression. Results from the longitudinal follow-up of the National Psychiatric Morbidity Survey. Br J Psychiatry 2005; 187: 544-51.

4 Boschloo L, Vogelzangs $\mathrm{N}$, Smit JH, Van den Brink W, Veltman DJ, Beekman AT, et al. Comorbidity and risk indicators for alcohol use disorders among persons with anxiety and/or depressive disorders: findings from the Netherlands Study of Depression and Anxiety (NESDA). J Affect Disord 2011; 131: 233-42.

5 Hasin DS, Stinson FS, Ogburn E, Grant BF. Prevalence, correlates, disability, and comorbidity of DSM-IV alcohol abuse and dependence in the United States: results from the National Epidemiologic Survey on Alcohol and Related Conditions. Arch Gen Psychiatry 2007; 64: 830-42.

Lynn Boschloo, PhD, Department of Psychiatry and EMGO Institute for Health and Care Research, VU University Medical Centre, Amsterdam, and Department of Psychiatry, University Medical Centre, Hanzeplein 1, Groningen, The Netherlands; Email: I.boschloo@umcg.nl; Nicole Vogelzangs, PhD, Department of Psychiatry and EMGO Institute for Health and Care Research, VU University Medical Centre, Amsterdam; Wim van den Brink, PhD, MD, Department of Psychiatry, Academic Medical Centre, University of Amsterdam, Amsterdam; Johannes H. Smit, PhD, Department of Psychiatry and EMGO Institute for Health and Care Research, VU Department of Psychiatry and EMGO Institute for Health and Care Research, VU University Medical Centre, Amsterdam; Dick J. Veltman, PhD, MD, Department of
Psychiatry, VU University Medical Centre, Amsterdam; Aartjan T. F. Beekman, PhD MD, Department of Psychiatry and EMGO Institute for Health and Care Research, VU University Medical Centre, Amsterdam; Brenda W. J. H. Penninx, PhD, Department of Psychiatry and EMGO Institute for Health and Care Research, VU University Medica Centre, Amsterdam, Department of Psychiatry, University Medical Centre, Groningen, and Department of Psychiatry, Leiden University Medical Centre, The Netherlands

doi: 10.1192/bjp.201.4.326a

\section{Depression in the workplace: what is depression?}

As a former consultant occupational psychiatrist to the Metropolitan Police and a contributor to the Black \& Frost report, ${ }^{1}$ I would like to point to the basic flaw in the paper by Gilbody et al. ${ }^{2}$

In Gilbody et al's account of 'depression in the workplace', they take 'depression' as a given, requiring no further explanation, yet there is currently no psychiatric category so bloated and subject to overdiagnosis. My experience of 900 assessments of individuals at the Metropolitan Police, $99 \%$ of whom were certificated as being 'off sick' with depression or one of the other 'common mental disorders', showed that the psychiatrisation of non-specific symptoms led routinely to unduly prolonged sickness absence and thus unnecessary disability. ${ }^{3}$ Sickness absence was strongly associated with workplace problems and other situational stresses: dislocation to a psychiatric arena frequently paralysed the practical problem-solving that would have normalised the situation in timely fashion. I found that National Health Service (NHS) mental health services were disconnected from the occupational aspects of patients' lives, and saw diagnosis and treatment as having a life of its own. Antidepressants and periodic out-patient appointments did not seem a model that got many people back to work. Naturally, I support Gilbody et al's advocacy of closer relations with the workplace but NHS services will continue to be as much part of the problem as of the solution without a review of the narrowly biomedical culture of much psychiatric practice.

Last, Gilbody et al see hope in the Layard Improving Access to Psychological Therapies (IAPT) programme. It is laughable hubris for the profession to imagine that large numbers of very heterogeneous people, many of whom have been out of the workplace for years, can be gathered together under the rubric of 'common mental disorder' and restored to full productivity via a few sessions of talk therapy.

1 Black C, Frost D. Health at Work - An Independent Review of Sickness Absence. TSO (The Stationery Office), 2011.

2 Gilbody S, Bower P, Rick J. Better care for depression in the workplace: integrating occupational and mental health services. Br J Psychiatry 2012; 200: $442-3$

3 Summerfield D. Metropolitan Police blues: protracted sickness absence, ill health retirement, and the occupational psychiatrist. BMJ 2011; 342: 950-2.

Derek Summerfield, Consultant Psychiatrist, Institute of Psychiatry, King's College London, UK. Email: Derek.Summerfield@slam.nhs.uk

doi: 10.1192/bjp.201.4.327

\section{Severe mental illness in prisoners worldwide}

We read with interest the review article by Fazel \& Seewald. ${ }^{1}$ The authors conclude that severe mental illness is more prevalent in prisoners in low- and middle-income (LAMI) countries than in high-income countries. This may be related to fewer opportunities and services for diverting offenders to health services, a stronger relationship between mental illness and criminality, and different sociocutural factors (e.g. poorer legal representation for the mentally ill in LAMI countries).

We examined in an earlier study the relationship between mental illness and criminality in pre-trial reported Antillean defendants in The Netherlands and The Netherlands Antilles. ${ }^{2}$ Defendants who are suspected to have a mental illness are examined pre-trial by a psychiatrist or psychologist both in The Netherlands and The Netherlands Antilles, before diversion to mental health services takes place. We found no significant difference in the prevalence of psychotic disorders among pre-trial reported Antillean suspects in The Netherlands and The Netherlands Antilles (14.4\% v. 15.1\%) and no significant difference in the prevalence of defendants deemed unaccountable due to a mental disorder $(3.9 \%$ v. $4.0 \%)$. There was, however, a much lower registered crime rate of Antilleans in The Netherlands Antilles than The Netherlands (11 v. 113 offences per year per 1000 persons; $P<0.001)$ and a much higher rate of pre-trial reports in The Netherlands Antilles than The Netherlands (74 v. 8 per 1000 Antillean defendants; $P<0.001$ ). Antilleans living in The Netherlands Antilles thus have a low crime rate and a high pre-trial report rate when indicted of a crime compared with Antilleans in The Netherlands, indicating that they are more frequently suspected to have a mental illness.

This finding is in line with the earlier formulated rule of thumb that the frequency of mental illness is higher in countries with low crime rates. ${ }^{3,4}$ Indeed, the LAMI countries included in Fazel \& Seewald's review have a combined prison population rate 